\title{
Gas Chromatography Coupled to Atmospheric Pressure Chemical lonization FT-ICR Mass Spectrometry for Improvement of Data Reliability
}

\author{
Theo Schwemer, ${ }^{\dagger \neq}$ Christopher P. Rüger, ${ }^{\dagger}$ Martin Sklorz, ${ }^{\star,+},{ }^{\prime}$ and Ralf Zimmermann ${ }^{\dagger \neq, \S}$ \\ †Joint Mass Spectrometry Centre / Chair of Analytical Chemistry, University of Rostock, 18051 Rostock, \\ Germany \\ $\ddagger$ HICE - Helmholtz Virtual Institute of Complex Molecular Systems in Environmental Health - Aerosols and \\ Health, 85764 Neuherberg, Germany, www.hice-vi.eu \\ §Joint Mass Spectrometry Centre / Cooperation Group Comprehensive Molecular Analytics, Helmholtz Zentrum \\ München, 85764 Neuherberg, Germany \\ * Corresponding author: martin.sklorz@uni-rostock.de
}

\section{Supporting Information}




\section{Table of Contents}

Table S-1. Sample acquisition and preparation

Table S-2. GC parameters

Table S-3. APCI source and FT-ICR MS parameters

Table S-4. Compound class distribution of fuels and particulate matter with applied KI correlation based on intensity weights

Table S-5. Polycyclic aromatic hydrocarbons used for calibration of retention index with corresponding Kováts Indices from NIST08 database

Figure S-1. Comparison of compound class distributions with and without applying KI correlation for compound filtering according to GC APCI FT-ICR analysis of diesel fuel (DF), particulate matter of diesel fuel (DF-PM), heavy fuel oil (HFO) and particulate matter of heavy fuel oil (HFO-PM)

Figure S-2. Intensity weighted DBE distribution of the major compound classes for GC APCI FT-ICR analyses with summed relative intensity of compound classes (standardized to summed intensity of all detected compounds) of a) DF - ultra low sulfur ( $\leq 10 \mathrm{ppm})$ diesel fuel according DIN EN 590; b) DF-PM - primary combustion particulate matter of diesel fuel; c) HFO - heavy fuel oil containing $2.7 \%$ sulfur, which is a representative fuel for ship operation outside of sulfur emission control areas; d) HFO-PM - primary combustion particulate matter of heavy fuel oil

Figure S-3. Carbon number vs. DBE plot of two abundant compound classes for all samples (diesel fuel (DF), particulate matter of diesel fuel (DF-PM), heavy fuel oil (HFO) and particulate matter of heavy fuel oil (HFO-PM)); left column are $\mathrm{CH}$ classes and right column are $\mathrm{CHO} 2$ classes

Figure S-4. Kendrick plot detected chromatographic features in heavy fuel oil (HFO) with (green) and without (blue) retention index correlation for filtering of assigned elemental compositions 
Table S-1: Sample acquisition and preparation

\begin{tabular}{|c|c|}
\hline engine & four stroke single-cylinder direct-injected ship diesel test engine \\
\hline used fuels & heavy fuel oil (HFO 180) and distillate fuel (DF) according to EN 590 \\
\hline $\begin{array}{l}\text { sample } \\
\text { acquisition }\end{array}$ & $\begin{array}{l}\text { engine exhaust gas was collected for four hours on quartz fiber filter discs } \\
\text { after cooling the exhaust gas to near room temperature by appropriate } \\
\text { dilution with compressed air }\end{array}$ \\
\hline $\begin{array}{l}\text { exhaust gas } \\
\text { dilution ratios }\end{array}$ & 1:10 for diesel fuel and 1:40 for heavy fuel oil \\
\hline $\begin{array}{l}\text { sample } \\
\text { preparation } \\
\text { particulate matter }\end{array}$ & $\begin{array}{l}\text { the filters were extracted twice with } 25 \mathrm{ml} \text { methanol/dichloromethane }(50 / 50 \text {, } \\
\mathrm{v} / \mathrm{v} \text { ) for } 15 \mathrm{~min} \text { using an ultrasonic bath. Both extractions were combined and } \\
\text { filtered via a } 0.2 \mu \mathrm{m} \text { PTFE-membrane filter. An aliquot of } 5 \mathrm{~mL} \text { was } \\
\text { concentrated to about } 0.1 \mathrm{~mL} \text { by applying a stream of pure nitrogen at room } \\
\text { temperature }\end{array}$ \\
\hline $\begin{array}{l}\text { sample } \\
\text { preparation fuel }\end{array}$ & $\begin{array}{l}\text { 1:100 dilution in a mixture of methanol/dichloromethane }(50 / 50, \mathrm{v} / \mathrm{v}) \text { and } \\
\text { directly analyzed without further treatments }\end{array}$ \\
\hline
\end{tabular}


Table S-2: GC parameters

\begin{tabular}{|l|l|}
\hline GC Type & CP 3800 (Agilent, former Varian Technologies, Palo Alto, CA, USA) \\
\hline injector & $\begin{array}{l}1079 \text { programmable temperature vaporizer injector equipped with a } \\
\text { ChromatoProbe device }\end{array}$ \\
\hline column & $\begin{array}{l}\text { HT5 column }(25 \mathrm{~m} \text { length } \mathrm{x} 0.53 \mathrm{~mm} \text { i.d. } \times 0.15 \mu \mathrm{m} \text { film thickness, } \\
\text { SGE Analytical Science, Australia) }\end{array}$ \\
\hline injection & splitless \\
\hline injection volume & $2 \mu \mathrm{L}$ \\
\hline injection program & $60^{\circ} \mathrm{C}(1 \mathrm{~min}) \rightarrow 300^{\circ} \mathrm{C}$ with $50^{\circ} \mathrm{C} / \mathrm{min}$ \\
\hline oven program & $40^{\circ} \mathrm{C}(6 \mathrm{~min}) \rightarrow 320^{\circ} \mathrm{C}$ with $10^{\circ} \mathrm{C} / \mathrm{min}$ \\
\hline flow rate & $\operatorname{constant~at~} 10 \mathrm{~mL} / \mathrm{min}$ of $99.999 \%$ Helium \\
\hline $\begin{array}{l}\text { temperature } \\
\text { transfer line }\end{array}$ & $320^{\circ} \mathrm{C}$ \\
\hline
\end{tabular}


Table S-3: APCI source and FT-ICR MS parameters

\begin{tabular}{|c|c|}
\hline source & $\begin{array}{l}\text { atmospheric pressure chemical ionization source with a } \\
\text { specialized gas phase } \text { inlet }^{16}\end{array}$ \\
\hline mass spectrometer & $\begin{array}{l}\text { Apex Qe Series II FT-ICR MS system (Bruker Daltonics, } \\
\text { Billerica, MA, USA / Bruker Daltonics GmbH, Bremen, } \\
\text { Germany) }\end{array}$ \\
\hline magnet & 7 T magnet (Bruker Biospin, France) \\
\hline operating mode & positive mode \\
\hline needle current & $3 \mu \mathrm{A}$ \\
\hline source temperature & $320^{\circ} \mathrm{C}$ \\
\hline sheath gas & nitrogen with a flow rate of $2 \mathrm{~L} / \mathrm{min}$ \\
\hline auxiliary gas & nitrogen with a flow rate of $0.6 \mathrm{~L} / \mathrm{min}$ \\
\hline dry gas & nitrogen with a flow rate of $2 \mathrm{~L} / \mathrm{min}$ \\
\hline nebulizer gas & nitrogen with a pressure of $3 \times 10^{5} \mathrm{~Pa}$ \\
\hline acquisition rate & approximately $0.6 \mathrm{~Hz}$ \\
\hline resolution at $\mathbf{m} / \mathbf{z} 300$ & 200000 \\
\hline mass range & $100-700 \mathrm{Da}$ \\
\hline acquisition software & Bruker Compass Data Analysis 4.0 SP 5 software package \\
\hline transient operations & $\begin{array}{l}\text { zero-filled, square sine-bell apodization and fast Fourier } \\
\text { transformation into frequency domain applied }\end{array}$ \\
\hline mass-calibration & $\begin{array}{l}\text { precalibration with fatty acid methyl ester mix (C10 - C24; } \\
\text { Supelco, Bellefonte, PA, USA) and internal calibration with an } \\
\text { averaged mass spectrum of the chromatogram, using well known } \\
\text { PAH signals of the samples and column bleed covering a m/z- } \\
\text { range of } 128 \text { to } 540\end{array}$ \\
\hline $\begin{array}{l}\text { standard deviation of } \\
\text { linear mass calibration }\end{array}$ & $<0.3 \mathrm{ppm}$ \\
\hline $\begin{array}{l}\text { used Signal-to-noise } \\
\text { ratio for export to } \\
\text { MATLAB }\end{array}$ & $\geq 9$ \\
\hline
\end{tabular}


Table S-4: Compound class distribution of fuels and particulate matter with applied KI correlation based on intensity weights

\begin{tabular}{|l|c|c|c|c|}
\hline $\begin{array}{l}\text { compound } \\
\text { classes }\end{array}$ & diesel fuel & heavy fuel oil & $\begin{array}{c}\text { particulate matter of } \\
\text { diesel fuel }\end{array}$ & $\begin{array}{c}\text { particulate matter of } \\
\text { heavy fuel oil }\end{array}$ \\
\hline CH & $75.4 \%$ & $34.8 \%$ & $15.5 \%$ & $48.8 \%$ \\
\hline CHO & $3.9 \%$ & $7.8 \%$ & $11.6 \%$ & $11.5 \%$ \\
\hline CHO2 & $19.4 \%$ & $13.9 \%$ & $61.4 \%$ & $17.0 \%$ \\
\hline CHO3 & $1.0 \%$ & $1.8 \%$ & $6.1 \%$ & $2.3 \%$ \\
\hline CHO4 & $0.0 \%$ & $0.0 \%$ & $0.5 \%$ & $0.5 \%$ \\
\hline CHN & $0.2 \%$ & $11.0 \%$ & $0.6 \%$ & $0.8 \%$ \\
\hline CHNO & $0.0 \%$ & $0.1 \%$ & $2.4 \%$ & $1.4 \%$ \\
\hline CHS & $0.0 \%$ & $23.6 \%$ & $0.1 \%$ & $15.6 \%$ \\
\hline CHSO & $0.0 \%$ & $6.3 \%$ & $0.0 \%$ & $0.2 \%$ \\
\hline others & $1.1 \%$ & $0.7 \%$ & $2.8 \%$ & $2.0 \%$ \\
\hline
\end{tabular}


Table S-5: Polycyclic aromatic hydrocarbons used for calibration of retention index with corresponding Kováts Indices from NIST08 database

\begin{tabular}{|l|l|l|l|r|}
\hline number & substance & $\begin{array}{l}\text { elemental } \\
\text { composition }\end{array}$ & $\begin{array}{l}\text { protonated } \\
\boldsymbol{m} / \boldsymbol{z} \text { [Da] }\end{array}$ & $\begin{array}{l}\text { Kováts } \\
\text { Index [IU] }\end{array}$ \\
\hline 1 & Naphthalene & $\mathrm{C}_{10} \mathrm{H}_{8}$ & 129.06988 & 1187.3 \\
\hline 2 & Acenaphthylene & $\mathrm{C}_{12} \mathrm{H}_{8}$ & 153.06988 & 1460.8 \\
\hline 3 & Acenaphthene & $\mathrm{C}_{12} \mathrm{H}_{10}$ & 155.08553 & 1479.9 \\
\hline 4 & Fluorene & $\mathrm{C}_{13} \mathrm{H}_{10}$ & 167.08553 & 1582.9 \\
\hline 5 & Phenanthrene & $\mathrm{C}_{14} \mathrm{H}_{10}$ & 179.08553 & 1775.0 \\
\hline 6 & Pyrene & $\mathrm{C}_{16} \mathrm{H}_{10}$ & 203.08553 & 2111.7 \\
\hline 7 & Chrysene & $\mathrm{C}_{18} \mathrm{H}_{12}$ & 229.10118 & 2458.5 \\
\hline 8 & Benzo[a]pyrene & $\mathrm{C}_{20} \mathrm{H}_{12}$ & 253.10118 & 2765.1 \\
\hline 9 & Dibenz(gh)anthracene & $\mathrm{C}_{22} \mathrm{H}_{14}$ & 279.11683 & n.a. \\
\hline 10 & Benzo[ghi]perylene & $\mathrm{C}_{22} \mathrm{H}_{12}$ & 277.10118 & 3151.6 \\
\hline 11 & Coronene & $\mathrm{C}_{24} \mathrm{H}_{12}$ & 301.10118 & n.a. \\
\hline
\end{tabular}



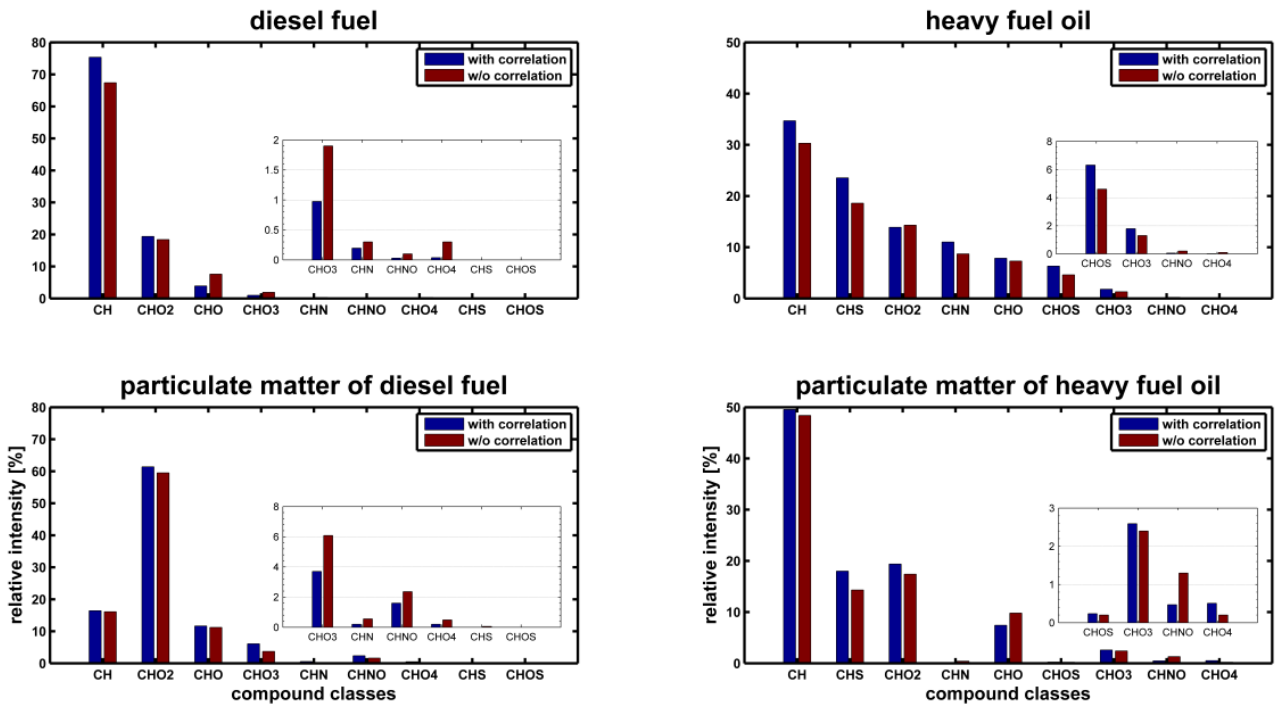

Figure S-1. Comparison of compound class distributions with and without applying KI correlation for compound filtering according to GC APCI FT-ICR analysis of diesel fuel (DF), particulate matter of diesel fuel (DF-PM), heavy fuel oil (HFO) and particulate matter of heavy fuel oil (HFO-PM) 


\section{diesel fuel}

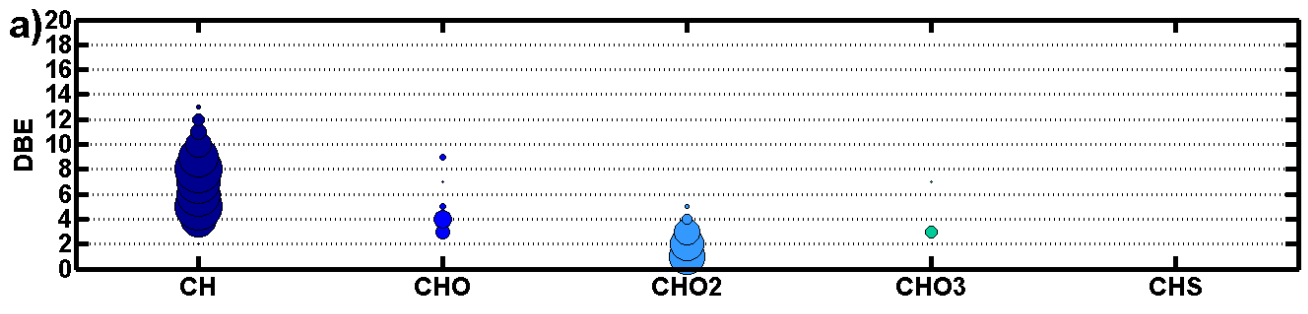

particulate matter of diesel fuel
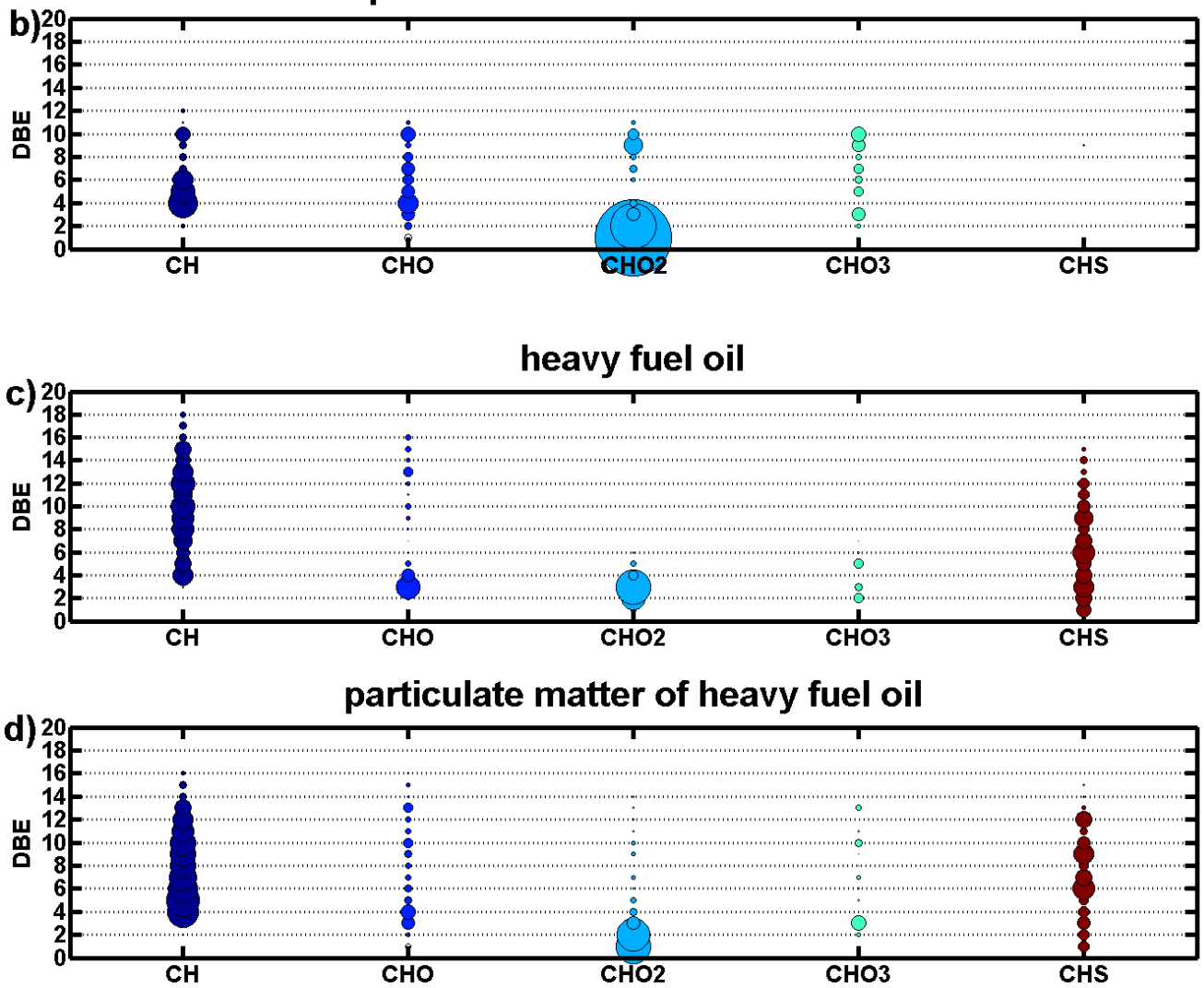

Figure S-2. Intensity weighted DBE distribution of the major compound classes for GC APCI FT-ICR analyses with summed relative intensity of compound classes (standardized to summed intensity of all detected compounds) of a) DF - ultra low sulfur ( $\leq 10 \mathrm{ppm}$ ) diesel fuel according DIN EN 590; b) DF-PM - primary combustion particulate matter of diesel fuel; c) HFO - heavy fuel oil containing $2.7 \%$ sulfur, which is a representative fuel for ship operation outside of sulfur emission control areas; d) HFO-PM - primary combustion particulate matter of heavy fuel oil 

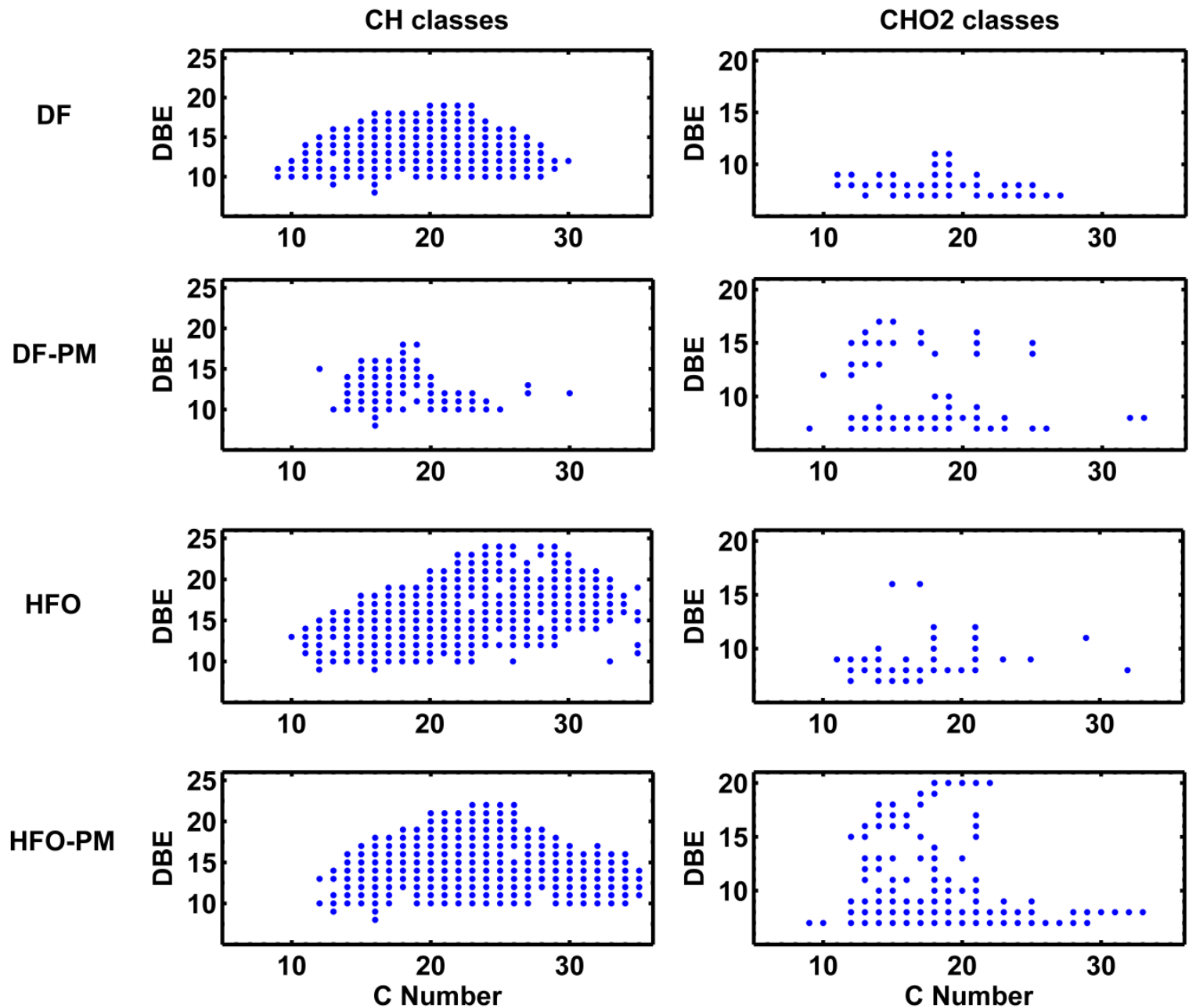

Figure S-3. Carbon number vs. DBE plot of two abundant compound classes for all samples (diesel fuel (DF), particulate matter of diesel fuel (DF-PM), heavy fuel oil (HFO) and particulate matter of heavy fuel oil (HFO$\mathrm{PM})$ ); left column are $\mathrm{CH}$ classes and right column are $\mathrm{CHO} 2$ classes 


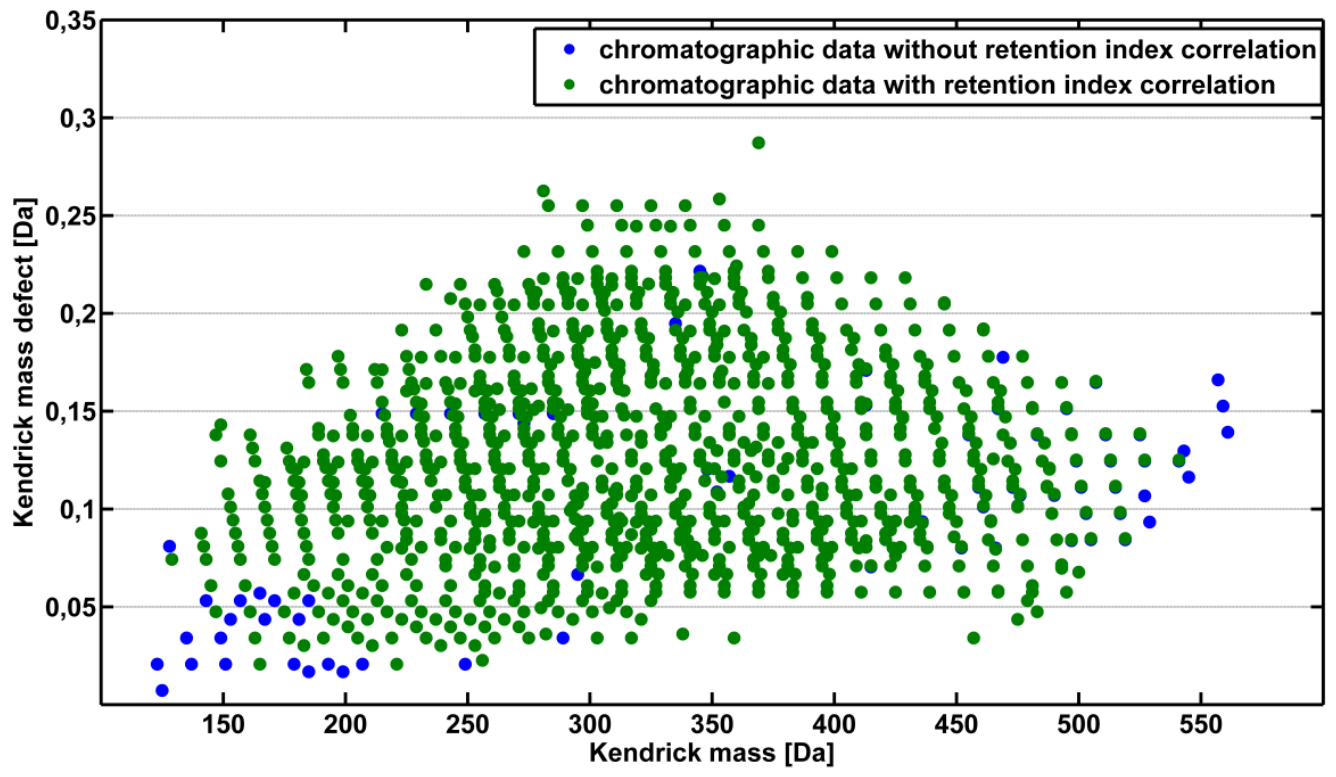

Figure S-4. Kendrick plot detected chromatographic compounds in heavy fuel oil (HFO) with (green) and without (blue) retention index correlation for filtering of assigned elemental compositions 